\title{
Simulation and Experiment of Underwater Target Active Electromagnetic Detection Based on SLF/ELF Artificial Source
}

\author{
Zongyang Shi ${ }^{1}{ }^{*}$, Yiyu Zhao ${ }^{1}$, Lichao Ma ${ }^{1}$, Xueming Peng ${ }^{1}$, Yike Xu ${ }^{1}$, Shurong Guo ${ }^{1}$, Meng Wang ${ }^{2}$, \\ Yuhong Wang ${ }^{3}$
}

${ }^{1}$ The Fourth Research Department, Beijing Institute of Mechanical Equipment, Beijing, China

${ }^{2}$ School of Geophysics and Information technology, China University of Geosciences (Beijing), Beijing, China

${ }^{3}$ Ocean College, Zhejiang University, Zhoushan, China

\section{Email address:}

shizongyang13@mails.ucas.ac.cn (Zongyang Shi), zhyznd@163.com (Yiyu Zhao),wangmeng@cugb.edu.cn (Meng Wang), wyhong@zju.edu.cn (Yuhong Wang)

${ }^{*}$ Corresponding author

\section{To cite this article:}

Zongyang Shi, Yiyu Zhao, Lichao Ma, Xueming Peng, Yike Xu, Shurong Guo, Meng Wang, Yuhong Wang. Simulation and Experiment of Underwater Target Active Electromagnetic Detection Based on SLF/ELF Artificial Source. Science Discovery. Vol. 9, No. 2, 2021 , pp. 58-67. doi: 10.11648/j.sd.20210902.17

Received: March 3, 2021; Accepted: April 15, 2021; Published: April 26, 2021

\begin{abstract}
In view of the detection performance constraints brought by the further application of demagnation and noise reduction technology to traditional sonar and magnetic anomaly detection technology, in order to explore the possibility and detection mode of active electromagnetic (EM) detection technology applied to underwater large target detection and positioning, the active EM detection method for underwater targets based on SLF/ELF artificial EM field source is established in this paper, and the active EM detection method of underwater target is modeled and simulated by Ansys Maxwell, the magnetic anomaly field induced by underwater target is calculated and analyzed. Then, the key parameters of the underwater target active EM detection system are listed based on the simulation, and a underwater target active EM detection prototype has been developed. Finally, the simulation results and prototype parameters of the active EM detection method of underwater targets are verified by the scaled ratio experiment. The results show that the parameters of prototype system designed according to the simulation results are correct and effective, and the active EM detection technology based on SLF/ELF can be applied to the underwater large target detection.
\end{abstract}

Keywords: Active Underwater Target Detection, Simulation Model bAsed on Ansys, Underwater Target Detection Experiment, Magnetic Detection

\section{基于SLF/ELF人工源的水下目标主动电磁探测仿真与实验}

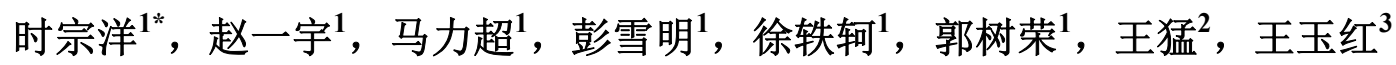

1第四研究室, 北京机械设备研究所, 北京, 中国

2中国地质大学 (北京) 地球物理与信息技术学院, 北京, 中国

3浙江大学海洋学院, 舟山, 中国

邮箱 shizongyang13@mails.ucas.ac.cn（时宗洋）, zhyznd@163.com（赵一宇）, wangmeng@cugb.edu.cn（王猛）, wyhong@zju.edu.cn(王玉红) 
摘要：针对当前及未来水下大型目标进一步应用消磁降噪技术给传统声呐及磁异常探测技术探测性能提升带来的瓶颈 约束问题, 为探索主动电磁探测技术应用于水下大型目标探测定位的可能性及探测模式, 本论文建立了基于超低频 (SLF)/极低频(ELF)人工电磁场源的水下目标主动电磁探测方法, 通过Ansys Maxwell对水下目标主动电磁探测方法进行 建模仿真, 分析了目标磁异常场; 然后, 基于仿真结果给出水下目标主动电磁探测系统关键参数, 并研制了一套水下 目标主动电磁探测样机; 最后, 通过缩比实验对水下目标主动探测方法仿真结果及样机参数进行了初步验证。结果表 明, 根据仿真结果设计的缩比样机系统参数正确有效, 基于SLF/ELF的主动电磁探测技术能够应用于水下大型目标的 探测。

关键词: 水下目标主动探测, Ansys建模仿真，水下目标探测实验，磁探测

\section{1. 引言}

水下大型目标探测一直是关系国家资源、安全等重 要技术领域, 传统上由于光、电等物理量在水下传播困 难, 声呐是水下探测的主要技术手段。声呐分为主动式 声呐和被动式声呐两种, 主动式声呐通过主动发送声波 并检测回波实现对目标的定位, 由于需要发送大功率声 波, 因此往往在有效检测范围外, 就将自身位置暴露给 了目标; 被动声呐通过监听目标引起的特征音频信号, 寻找目标的大致方位, 做不到对目标的精准定位。在这 一背景下, 发展非声探测技术成了函需解决的任务。非 声探测手段主要有红外探测、激光探测、电磁探测等, 由于一般来说, 构成水下大型目标的材料中不可避免包 含铁磁性材料, 因此电磁探测方法脱颖而出, 成为最具 有潜力的技术发展方向 $[1,2]$ 。

电磁探测技术原本是在地质勘探领域发展起来的 一种探测技术, 从原理上, 分为主动式和被动式探测两 大类。将被动式电磁探测技术用于水下探测, 早在 1918 年, 美国就进行了船只拖曳式的探测实验。主动式电磁 探测即结合低频人工场源的一种电磁探测方式, 目前研 究仍然集中在地质勘探上, 从地质勘探的结果上来说相 对于被动式探测，在分辨率，探测速度等方面具备一定 的技术优势 [3, 4], 因而有必要将这一探测技术应用于 水下目标探测领域, 希望能够得到优于被动磁异探测的 效果。

首先从原理上建立基于 $\mathrm{SLF} / \mathrm{ELF}$ 人工源的水下目 标电磁探测方法的理论, 从 Maxwell 方程出发, 研究 海水中的电磁传播特性, 结合仿真数据, 找到一种可能 的探测模式。为验证这一探测模式, 搭建了实验平台和 实验环境, 开展了水下探测相关实验。从实验结果上看, 达到了预期的探测效果, 因此说明该方法在水下大型目 标探测方面具备一定的研究价值。

\section{2. 基本理论}

海水环境中同样条件下, 水下水平电偶极天线较水 下垂直电偶极天线从海水中辐射进入空气中的电磁场 强度更具优势, 且水下水平磁偶极天线较水下垂直磁偶 极天线从海水中辐射进入空气中的电磁场强度更具优
势[5-7，9]。综合考虑四种天线辐射模式辐射性能和实 际实验的设计实施难易程度, 本论文选择以水下水平电 性天线作为电磁辐射天线。

\section{1. 海水中水平电性天线辐射的电磁场}

\subsection{1. 典型层状海洋模型}

典型层状海洋模型包含空气层、海水层和海底层, 其 中海底层可根据海底不同深度电导率变化分成多层海底。 考虑仿真模型的一般性, 本论文中所指的层状海洋模型中 的海底为均匀半空间。

海水层深度为 $d_{1}$, 空气-海水分界面与海水-海底分界 面互相平行, 水平方向上无限延伸, 空气、海水和海底的 介电参数分别为 $\sigma_{i} 、 \varepsilon_{i}, i=0,1,2$, 其中 $i=0$ 表示空气层, $i=1$ 表示海水层。真空磁导率为 $\mu_{0}$, 相对磁导率 $\mu_{r}$ 设置为 1 。 $\mathrm{AB}$ 表示电性天线首尾, 天线长度为 $L$, 天线电极矩为 $P=I L$, 天线中点位于原点 $O$ 正下方, 目标所在深度为 $H$, 距离海底深度为 $h$, 各层厚度为 $d_{i}$ 。笛卡尔坐标系正 $z$ 方 向垂直水平面向下, $o-x y z$ 满足右手螺旋定则。假设任意 姿态电性天线 (见图1) 在 $X O Y$ 平面内的投影与 $x$ 轴夹角 为 $\theta$, 天线与 $X O Y$ 平面的夹角为 $\phi$ 。

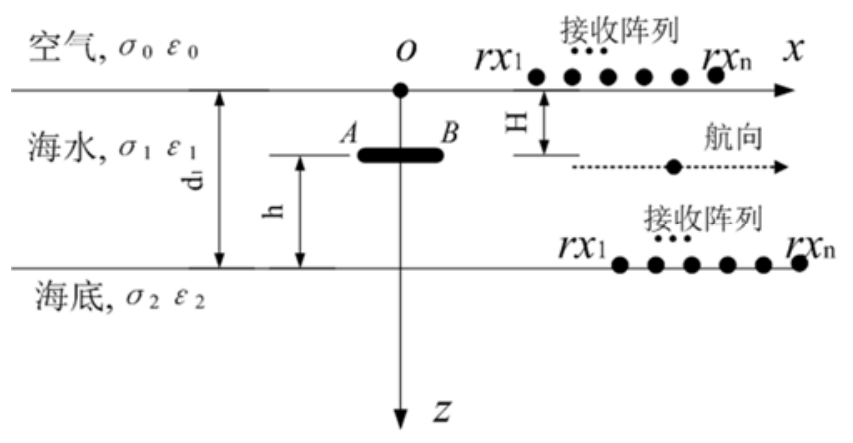

图1 层状海洋模型水平电偶极天线示意图。

\subsection{2. 水平电偶极天线辐射电磁场}

层状海洋模型下偶极天线辐射电磁场的推导基于以 下假设 $[1,8,11,13,15]$ :

a) 满足准静态近似条件, 频率应小于 $100 \mathrm{kHz}$;

b) 准静态近似下忽略海水和海底环境的位移电流, 波 数为 $k_{i}^{2}=-i \omega \mu_{0} \sigma_{i}, i=1,2$ 。空气中仅存在位移电 流, 约定电导率为 $\sigma_{0}=i \omega \varepsilon_{0}$, 波数为 $k_{0}^{2}=\omega^{2} \mu_{0} \varepsilon_{0}$; 
c) 空气、海水及海底媒介各向同性, 参数与时间、温 度及压强无关;

d) 准静态近似下, 认为媒介参数与频率无关, 磁导率 采用真空中的参数。

对于没有自由电荷的空间, 电性天线在空间中产生的 电磁场满足如下麦克斯韦方程组 [16, 18-19]。

$$
\left\{\begin{array}{l}
\nabla \times \mathrm{E}=j \mu \omega \mathrm{H} \\
\nabla \times \mathrm{H}=(\sigma-j \varepsilon \omega) \mathrm{E} \\
\nabla \cdot \mathrm{H}=0 \\
\nabla \cdot \mathrm{E}=0
\end{array}\right.
$$

定义电矢量为 $\mathbf{A}$, 满足 $\mathrm{H}=\nabla \times \mathrm{A}$ 。由式得到电场与电 矢位关系,

$$
\mathrm{E}=j \mu \omega \mathrm{A}-\nabla U
$$

其中 $U$ 为标量位, 满足 $U=-\frac{1}{\sigma} \nabla \cdot \mathrm{A}$ 。

层状海洋模型下水平电偶极天线辐射电磁场具有对 称性 [17], 其电矢量位 $A$ 仅包含沿偶极矩方向的分量 $A_{x}$ 和 沿垂直海水-空气界面的分量 $\mathrm{A}_{\mathrm{z}}$ 。并且电矢量位、电场及 磁场应满足下面条件:

a) 整个空间中, 除发射源位置外，矢量位 $\mathbf{A}$ 处处为有 限值, 且在无穷远处, 矢量位为零, 即 $\mathrm{A}(\mathrm{r} \rightarrow \infty) \rightarrow 0$;

b) 各层分解面上, 电场和磁场的切向分量连续。

根据边界条件约束, 电场 $E$ 、磁场 $H$ 与矢量为 $A$ 和 标量位 $U$ 之间的关系, 采用分离变量法可得到各层中矢量 位各分量的解。

空气中电磁场各分量频域计算式为:

$$
\begin{aligned}
& \left\{\begin{array}{l}
E_{x 0}=\frac{I d l}{4 \pi \sigma_{0}} \int_{0}^{\infty}\left[k_{0}^{2} C_{0}+\left(B_{0} m_{0}-C_{0}\right) m^{2} \cos ^{2} \phi\right] e^{m_{0} z} J_{0}(m r) d m \\
+\frac{I d l}{4 \pi \sigma_{0}} \int_{0}^{\infty}\left(B_{0} m_{0}-C_{0}\right) m e^{m_{0} z} \frac{\sin ^{2} \phi-\cos ^{2} \phi}{r} J_{1}(m r) d m \\
E_{y 0}=\frac{I d l}{4 \pi \sigma_{0}} \sin \phi \cos \phi \int_{0}^{\infty}\left(B_{0} m_{0}-C_{0}\right) m e^{m_{0} z}\left[m J_{0}(m r)-\frac{2}{r} J_{1}(m r)\right] d m \\
E_{z 0}=\frac{I d l}{4 \pi \sigma_{0}} \cos \phi \int_{0}^{\infty}\left(B_{0} m^{2}-C_{0} m_{0}\right) m e^{m_{0} z} J_{1}(m r) d m
\end{array}\right. \\
& \left\{\begin{array}{l}
H_{x 0}=\frac{I d l}{4 \pi} \sin \phi \cos \phi \int_{0}^{\infty} B_{0} m e^{m_{0} z}\left(m J_{0}(m r)-\frac{2}{r} J_{1}(m r)\right) d m \\
H_{y 0}=\frac{I d l}{4 \pi} \int_{0}^{\infty}\left(m_{0} C_{0}-m^{2} \cos ^{2} \phi B_{0}\right) e^{m_{0} z} J_{0}(m r) d m \\
-\frac{I d l}{4 \pi} \int_{0}^{\infty} B_{0} m e^{m_{0} z} \frac{\sin ^{2} \phi-\cos ^{2} \phi}{r} J_{1}(m r) d m \\
H_{z 0}=\frac{I d l}{4 \pi} \sin \phi \int_{0}^{\infty} C_{0} m e^{m_{0} z} J_{1}(m r) d m
\end{array}\right.
\end{aligned}
$$

其中, $I d l$ 表示电偶极源的电矩, $m$ 为积分变量, $r=\sqrt{x^{2}+y^{2}}$ 为收发距, $m_{i}=\sqrt{m^{2}-k_{i}^{2}}, i=0,1,2, \phi$ 为 观测点与电偶极源位置连线在XOY平面投影与 $x$ 轴的夹
角, $\sin \phi=\frac{y}{r}, \cos \phi=\frac{x}{r}$ 。积分核中的 $J_{i}(m r), i=0,1$ 代 表第 $i$ 阶贝塞尔函数。参数 $C_{i} 、 B_{i} 、 D_{i}$ 和 $E_{i}$ 为待定系数, 通过理论推导计算结果如下:

$$
\left\{\begin{array}{l}
C_{0}=\frac{m}{m_{1}} \frac{1-N_{01}}{e^{m_{1} H}} \frac{1-N_{21} e^{-2 m_{1} d_{1}} e^{2 m_{1} H}}{1-N_{21} N_{01} e^{-2 m_{1} d_{1}}} \\
B_{0}=B_{1}+E_{1} \\
C_{1}=\frac{m}{m_{1}} N_{21} \frac{e^{-2 m_{1} d_{1}}\left(N_{01} e^{-m_{1} H}-e^{m_{1} H}\right)}{1-N_{21} N_{01} e^{-2 m_{1} d_{1}}} \\
B_{1}=-\frac{P_{21} e^{-2 m_{1} d_{1}}}{m} \frac{e^{m_{1} H}+P_{01} e^{-m_{1} H}}{1-P_{01} P_{21} e^{-2 m_{1} d_{1}}}+\frac{m_{1}}{m^{2}} C_{1} \\
D_{1}=\frac{m}{m_{1}} N_{01} \frac{e^{-m_{1} H}-N_{21} e^{-2 m_{1} d_{1}} e^{m_{1} H}}{N_{21} N_{01} e^{-2 m_{1} d_{1}}-1} \\
E_{1}=\frac{P_{01} P_{21} e^{-2 m_{1} d_{1}}}{m} \frac{e^{m_{1} H}+P_{01} e^{-m_{1} H}}{1-P_{01} P_{21} e^{-2 m_{1} d_{1}}}+\frac{P_{01}}{m} e^{-m_{1} H}-\frac{m_{1}}{m^{2}} D_{1}
\end{array}\right.
$$

式中的 $N_{01} 、 N_{21} 、 P_{01}$ 和 $P_{21}$ 计算式如下:

$$
\left\{\begin{array}{c}
N_{01}=\frac{m_{0}-m_{1}}{m_{0}+m_{1}}, N_{21}=\frac{1-\frac{m_{1}}{m_{2}} R_{z}^{*}}{1+\frac{m_{1}}{m_{2}} R_{z}^{*}} \\
P_{01}=\frac{\sigma_{1} m_{0}-\sigma_{0} m_{1}}{\sigma_{1} m_{0}+\sigma_{0} m_{1}}, P_{21}=\frac{1-\frac{\rho_{1} m_{1}}{\rho_{2} m_{2}} Q_{2}^{*}}{1+\frac{\rho_{1} m_{1}}{\rho_{2} m_{2}} Q_{2}^{*}}
\end{array}\right.
$$

式中的 $R_{2}^{*}$ 和 $Q_{2}^{*}$ 的递推计算式为:

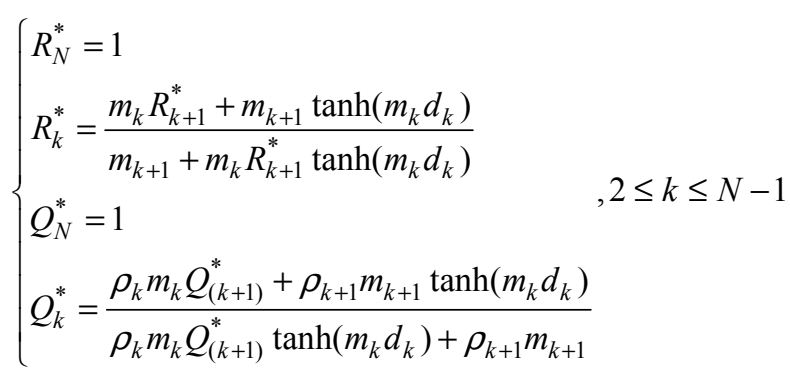

\subsection{3. 有限长度水平电性天线辐射电磁场}

典型层状海洋模型下有限长度水平电性天线辐射电 磁场的计算需要通过对天线长度进行积分获得。假设电性 天线 $l$ 位置的电偶极子在观测位置 $\vec{r}$ 处产生的响应（电场 或磁场）表示为 $f(p, \phi, \vec{l}, \vec{r})$, 其中 $f(\cdots)$ 可以表示辐射电 磁场 $E_{x} 、 E_{y} 、 E_{z} 、 B_{x} 、 B_{y} 、 B_{z}$ 的计算式。 $p=I d l, p$ 表示天线 $l$ 位置处的电偶极矩, $I$ 为天线中电流强度, $d l$ 表示 $l$ 位置的偶极子单位长度, $\vec{l}$ 和 $\vec{r}$ 分别为电偶极子天 线和观测点的相对于坐标原点的位置矢量。因此, 有限长 电性天线的电磁场计算式如下 


$$
\int_{L} f(p, \phi, \vec{l}, \vec{r}) d l
$$

实际应用中, 为实现加速计算同时保持计算精度可以 采用非均匀稀疏分割积分方法求解上式，相关方法参见 $[10,11]$ 。

\section{2. 海水中电磁场波传播特性}

为了在仿真建模过程中设置合理的天线辐射电磁波 基频和天线尺寸等参数, 需要对电磁波在海水中的传播特 性进行研究。根据电磁波在空气-海水界面传播规律, 电 磁波从海水中向空气传播时, 以近似垂直入射的形式穿透 海水-空气界面; 当电磁波从空气中向海水中传播时，无 论入射方向如何, 透射的电磁波均以近似垂直于海水-空 气界面形式传播[12-14, 19]。为增大主动探测模式下主动 电磁场对水下目标的感应范围, 需要尽可能的利用空气波 (侧面波, 路径为海水-空气-海水-目标) 或者海底导行波 (路径为海水-海底-海水-目标) 以实现衰减小和快速的感 应目标, 见图2。而无论是空气波还是海底导行波, 其传 播路径的最后从分界面到目标的电磁波近似为平面波, 为 此根据平面电磁波在海水中的衰减和传播规律确定论文 仿真设置的频率和天线长度。

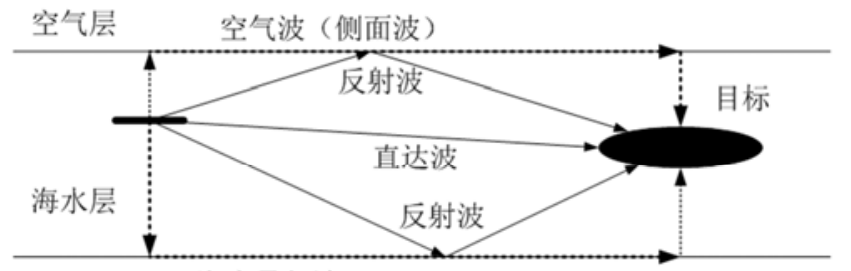

海底层

海底导行波

图2 SLF/ELF电磁波在海水中传播的多径相应。

\subsection{1. 海水中电磁波的特征参数}

根据平面电磁波在海水传播规律，其传播常数 $\gamma$ 、衰 减常数 $\alpha$ 和相移常数 $\beta$ 的计算式如下。其中, 海水媒介的磁 导率取真空磁导率, 海水相对介电常数为 81 , 电导率取 $4 \mathrm{~S} / \mathrm{m}[9]$ 。

$$
\begin{aligned}
& \gamma=\sqrt{j \omega \mu_{s} \sigma_{1}-\omega^{2} \mu_{s} \varepsilon_{1}} \\
& \alpha=\omega \sqrt{\frac{\mu_{s} \varepsilon_{1}}{2}\left(\sqrt{1+\frac{\sigma_{1}^{2}}{\omega^{2} \varepsilon_{1}^{2}}}-1\right)} \\
& \beta=\omega \sqrt{\frac{\mu_{s} \varepsilon_{1}}{2}\left(\sqrt{1+\frac{\sigma_{1}^{2}}{\omega^{2} \varepsilon_{1}^{2}}}+1\right)}
\end{aligned}
$$

电磁波在海水中的趋肤深度 $\delta$ 可根据衰减常数进行计 算。图3绘制电磁波趋肤深度与频率的关系, 可知为实现 百米级目标探测深度, 选用的 $0.01 \mathrm{~Hz} \sim \mathrm{n} \times 10 \mathrm{~Hz}$ 范围的频率。

$$
\delta=1 / \alpha \approx \sqrt{\frac{2}{\omega \mu_{s} \sigma_{1}}}
$$

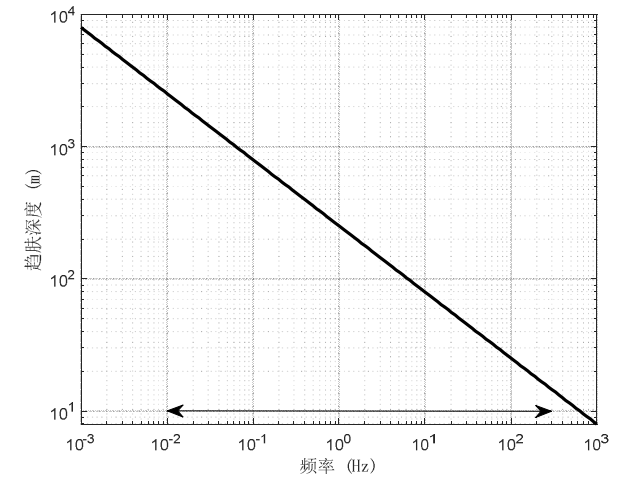

图3 海水中SLF/ELF电磁波的趋肤深度与频率的关系。

SLF/ELF电磁波在海水中的传播速度 $v$ 和波长 $\lambda$ 可以根 据相移常数计算。为实现几十米到百米的目标分辨率, 应 尽可能选择高频段, 这里可考虑选择 $10 \mathrm{~Hz} \sim 300 \mathrm{~Hz}$ 频段内 的电磁波。为尽可能提升水下天线的辐射功率, 天下尺寸 应与电磁波在海水媒介中波长的 $1 / 10$ 相当, 因此, 天线尺 寸选择 $9 \mathrm{~m} \sim 50 \mathrm{~m}$ 之间为较佳。

$$
\begin{aligned}
& v_{p}=\frac{\omega}{\beta} \\
& \lambda=\frac{v_{p}}{f}
\end{aligned}
$$

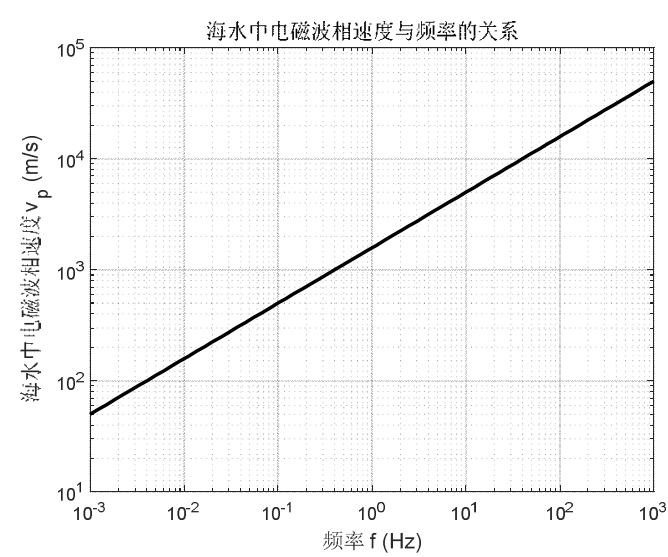

图4 海水中SLF/ELF电磁波波速与频率的关系。

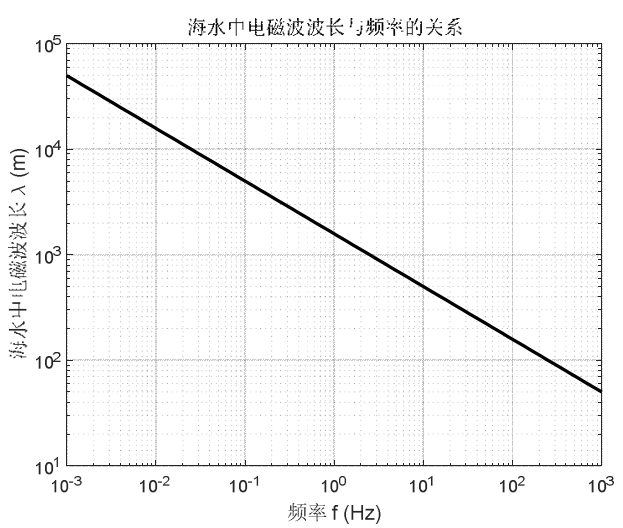

图5 海水中SLF/ELF电磁波波长与频率的关系。 


\subsection{2. 电磁波的频段选择}

综合考虑水下大型目标探测深度、目标分辨率等因素, 结合图3和图 5 关系图, 本论文仿真模型中选定电磁波频率 采用 $25 \mathrm{~Hz}$ 发射基频, 天线长度 $5 \mathrm{~m}$, 天线位于水下距离水 面 $10 \mathrm{~m}$ 的深度。

\section{3. 建模仿真与分析}

激励源加载、边界设置、仿真设置、观测设置（时 频域观测)、并对测点、测线和测面的仿真结果进行分析。

为对水下目标主动探测的可能性和效果进行分析, 建 立有无目标两种工况的仿真模型。两种工况下除目标有无 外, 其他条件相同。其中水下水平电性天线沿 $\mathrm{x}$ 轴方向, 目标轴向沿x方向。Ansys Maxwell进行建模仿真时设置参 数见表 1 。

表1 水下目标主动探测建模仿真参数列表。

\begin{tabular}{llll}
\hline \multirow{2}{*}{ 参数符号 } & 参数信息 & & \\
\cline { 2 - 4 } & 取值 & 单位 & 物理意义 \\
\hline$\varepsilon_{0}$ & $8.85 \times 10^{-12}$ & $\mathrm{~F} / \mathrm{m}$ & 真空介电常数 \\
$\mu_{0}$ & $4 \pi \times 10^{-7}$ & $\mathrm{H} / \mathrm{m}$ & 真空磁导率 \\
$\varepsilon_{\mathrm{sr}}$ & 81 & - & 海水相对介电常数 \\
$\sigma_{1}$ & 4 & $\mathrm{~S} / \mathrm{m}$ & 海水电导率 \\
$\mathrm{f}$ & 25 & $\mathrm{~Hz}$ & 天线辐射电磁场基频 \\
$\mathrm{L}$ & 5 & $\mathrm{~m}$ & 水下水平电性天线长度 \\
$\left(\mathrm{x}_{\mathrm{a}}, \mathrm{y}_{\mathrm{a}}, \mathrm{H}\right)$ & $(0,0,-10)$ & $\mathrm{m}$ & 水平电性天线所在位置 \\
$\mathrm{L}_{\mathrm{t}}$ & 100 & $\mathrm{~m}$ & 目标轴向长度 \\
$\mathrm{R}_{\mathrm{t}}$ & 10 & $\mathrm{~m}$ & 目标外径 \\
$(\mathrm{x}, \mathrm{y}, \mathrm{z})_{\mathrm{t}}$ & $(500,500,-100)$ & $\mathrm{m}$ & 目标所在位置 \\
$\sigma_{\mathrm{t}}$ & 1100000 & $\mathrm{~S} / \mathrm{m}$ & 目标电导率 \\
$\mu_{\mathrm{tr}}$ & 1 & - & 目标相对磁导率 \\
$\sigma_{\mathrm{sl}}$ & 0.01 & $\mathrm{~S} / \mathrm{m}$ & 海底电导率 \\
$\varepsilon_{\mathrm{slr}}$ & 1 & - & 海底相对介电常数 \\
$\mu_{\mathrm{slr}}$ & 1 & - & 海底相对磁导率 \\
\hline
\end{tabular}

\section{1. 水下目标主动探测仿真建模}

\subsection{1. 层状海洋模型仿真域}

图6给出了本论文建立的水下目标主动探测的仿真模 型域, 其中全局坐标系XOY的原点位于目标正上方的海水 平面上。水平面上方对应 $\mathrm{z}$ 轴为正, 海水中对应 $\mathrm{z}$ 轴为负。 海水域的长宽高大小为 $2 \mathrm{~km} \times 2 \mathrm{~km} \times 0.2 \mathrm{~km}$ 。模型中海水和海 底的网格剖分基于长度的体内部网格剖分。

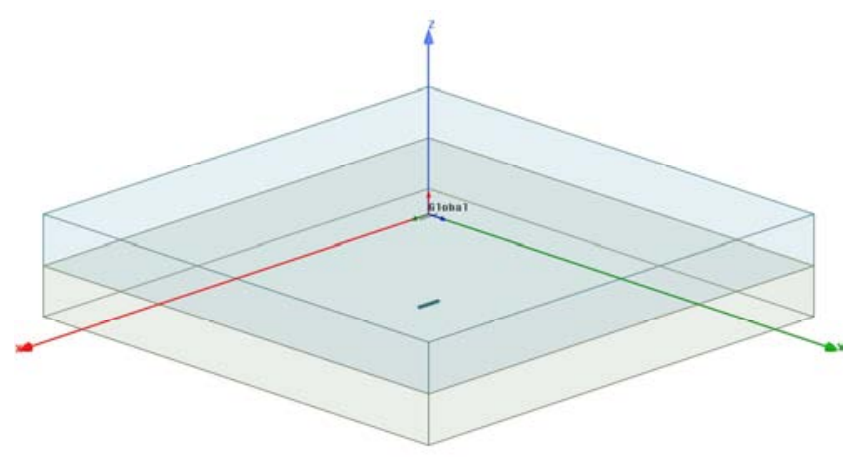

图6 层状海洋模型仿真计算域。
模型中目标的几何结构和网格剖分见图7, 其中目标 轴向长度 $100 \mathrm{~m}$, 外径 $10 \mathrm{~m}$ 。

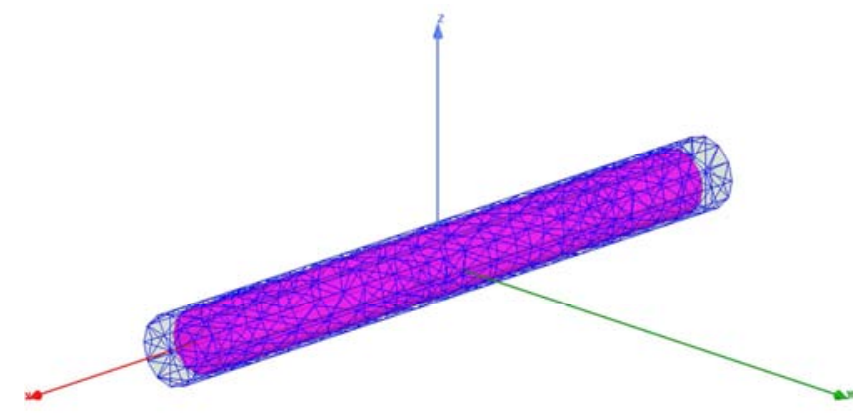

图7 圆柱形目标模型及网格剖分示意图。

\subsection{2. 仿真模型激励设置}

仿真模型激励添加基频 $25 \mathrm{~Hz}$, 占空比为 $50 \%$, 峰值电 流为 $5000 \mathrm{kA}$ 的方波电流, 设置仿真过程考虑海底、海水及 天线的涡流效应。

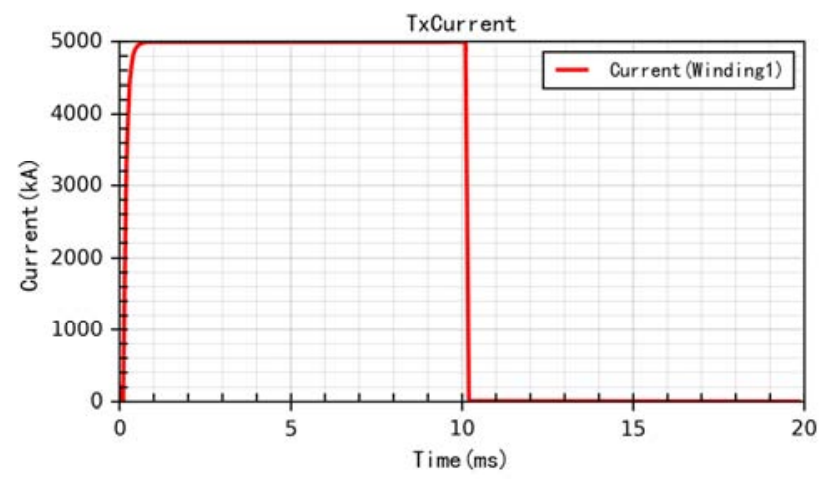

图8 水下目标电磁探测仿真激励波形。

仿真求解器可以选择Ansys Maxwell的时域(Transient) 求解模块或者频域(Eddy Current)求解模块, 本论文采用时 域求解模块, 计算分析脉冲期间或者脉冲间歇期间目标附 近海面磁场的分布特征及强度, 为提升仿真效率, 开启 Ansys仿真软件的GPU加速功能。

\subsection{3. 仿真模型观测设置}

仿真结果通过磁场各分量的测线和测面分布进行可 视化分析。测线编号及相关信息见表1。

表1 仿真观测测线设置。

\begin{tabular}{lll}
\hline \multirow{2}{*}{ 序号 } & 观测设置 & \\
\cline { 2 - 3 } & 测线编号 & 测线信息 \\
\hline 1 & LineXInSea & 目标正上方, 平行于X轴, 水下 $1 \mathrm{~m}$ \\
2 & LineXInAir_1m & 目标正上方, 平行于X轴, 水上 $1 \mathrm{~m}$ \\
3 & LineXInAir_100m & 目标正上方, 平行于X轴, 水上 $100 \mathrm{~m}$ \\
4 & LineYInSea & 目标正上方, 平行于Y轴, 水下 $1 \mathrm{~m}$ \\
5 & LineYInAir_1m & 目标正上方, 平行于Y轴, 水上 $1 \mathrm{~m}$ \\
6 & LineYInAir_100m & 目标正上方, 平行于Y轴, 水上 $100 \mathrm{~m}$ \\
\hline
\end{tabular}




\section{2. 水下目标主动探测仿真结果与分析}

\subsection{1. 测线仿真结果及分析}

图9-图12分别展示了总磁感应强度及各分量磁感应 强度沿X方向测线的分布图，观测时刻 $15 \mathrm{~ms}$, 其中目标位 于测线 $500 \mathrm{~m}$ 位置, 红色实线对应沿测线LineXInSea的分布 图; 棕色实线对应沿测线LineXInAir_1m的分布图; 蓝色 实线对应沿测线LineXInAir_100m的分布图。

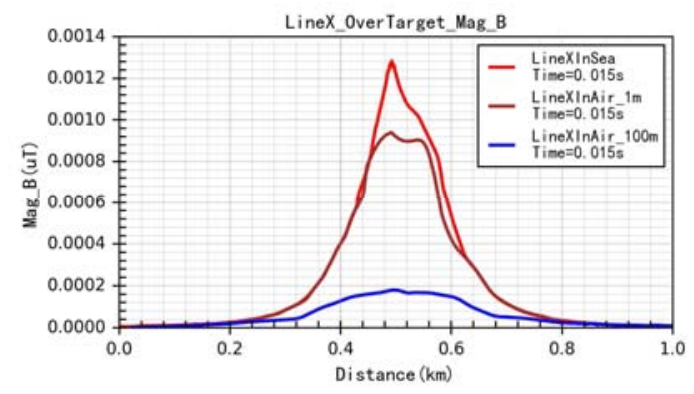

图9 总磁感应强度沿x方向测线分布图。

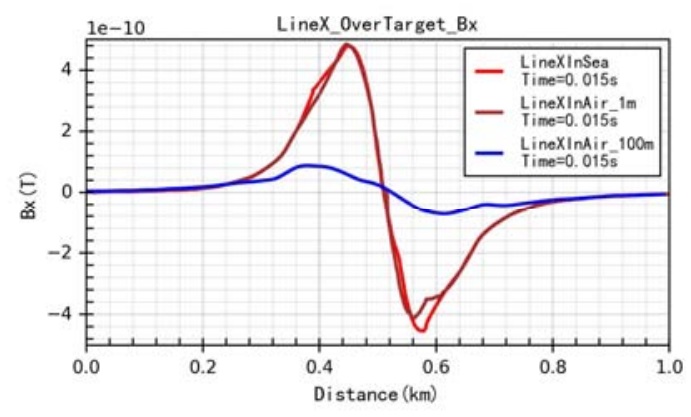

图10 $\mathrm{B}_{\mathrm{x}}$ 沿 $\mathrm{x}$ 方向测线分布图。

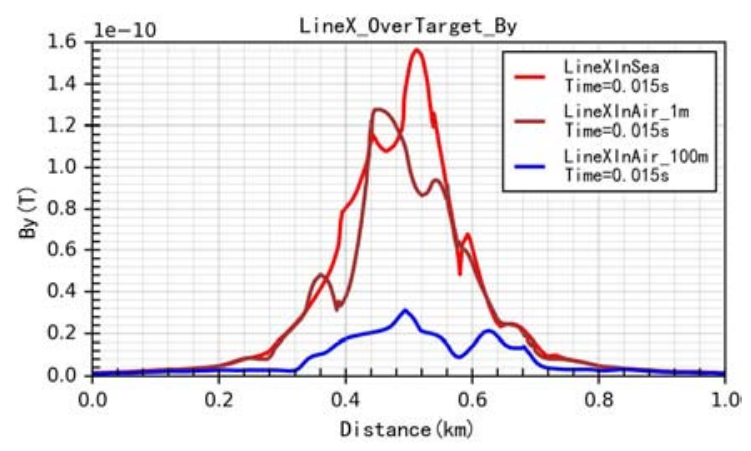

图11 B 沿x方向测线分布图。

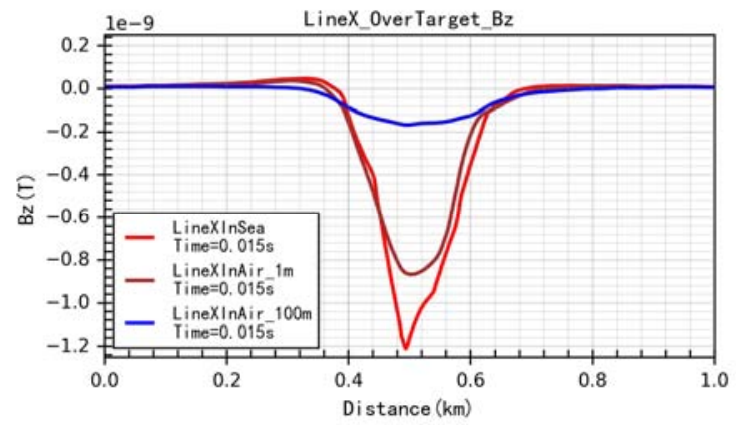

图12 $\mathrm{B}_{\mathrm{z}}$ 沿 $\mathrm{x}$ 方向测线分布图。
从测线分布特征可以发现: 测线经过目标正上方（此时 测线与目标轴向平行) 时, $\mathrm{B}_{\mathrm{x}}$ 在目标正上方出现信号极性反 转, 信号在目标位置两侧出现波峰和波谷, 测线分布关于目 标位置总体呈奇对称; $\mathrm{B}_{\mathrm{y}}$ 在目标正上方附近达到峰值, 测线 分布关于目标位置总体呈偶对称; $\mathrm{B}_{\mathrm{z}}$ 在目标正上方达到峰值, 测线分布关于目标位置总体呈偶对称; 总磁感应强度在目标 正上方达到峰值, 且关于目标位置总体呈偶对称。随着测线 位置高度的增加, 三轴响应信号幅值逐渐减小, 各分量磁感 应强度沿x方向测线分布峰值强度见表2。

表2 各分量磁感应强度沿x方向测线分布峰值强度。

\begin{tabular}{lllll}
\hline \multirow{2}{*}{ 测线编号 } & \multicolumn{4}{l}{ 各分量测线分布峰值强度(nT) } \\
\cline { 2 - 5 } & $\mathbf{B x}$ & $\mathbf{B y}$ & $\mathbf{B z}$ & MagB \\
\hline LineXInSea & 0.5 & 0.16 & 1.25 & 1.3 \\
LineXInAir_1m & 0.5 & 0.13 & 0.88 & 0.9 \\
LineXInAir_100m & 0.1 & 0.03 & 0.20 & 0.2 \\
\hline
\end{tabular}

图13-图16分别展示了总磁感应强度及各分量磁感应 强度沿Y方向测线的分布图, 观测时刻 $15 \mathrm{~ms}$, 其中目标位 于测线 $500 \mathrm{~m}$ 位置, 红色实线对应沿测线LineXInSea的分布 图; 棕色实线对应沿测线LineXInAir_1m的分布图; 蓝色 实线对应沿测线LineXInAir_100m的分布图。

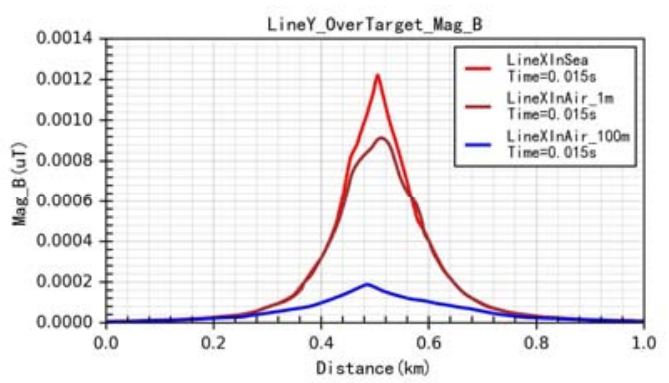

图13 总磁感应强度沿y方向测线分布图。

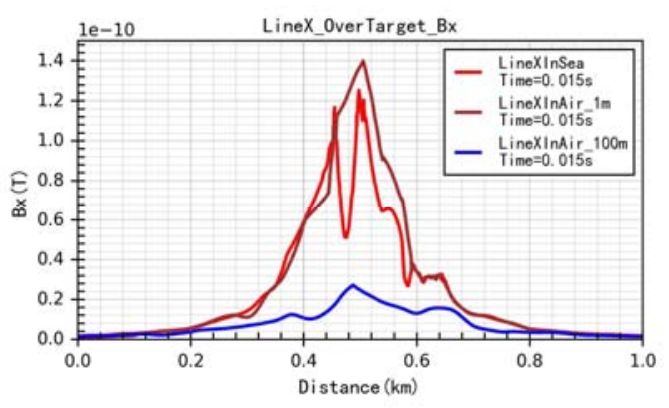

图14 $\mathrm{B}_{\mathrm{x}}$ 沿y方向测线分布图。

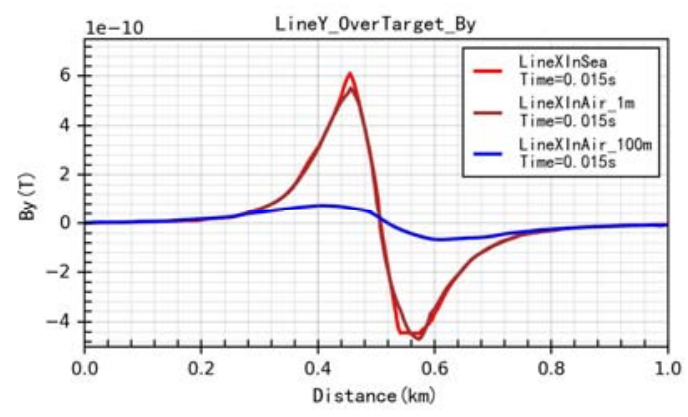

图15 By 沿y方向测线分布图。 


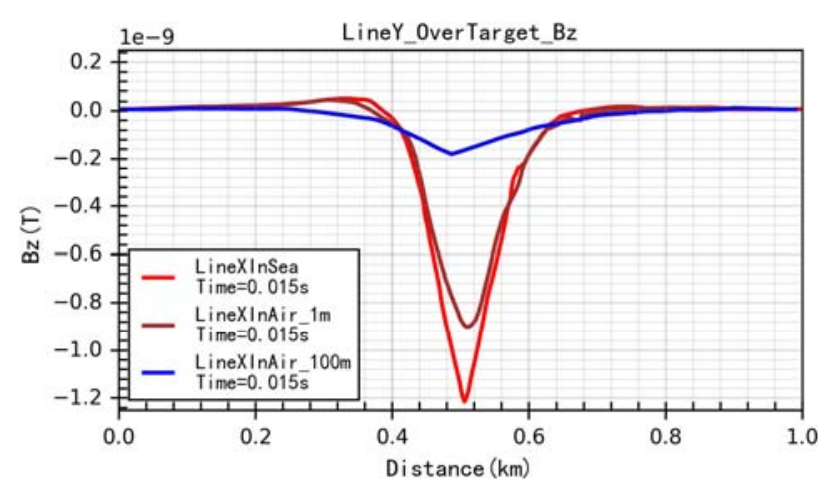

图16 $\mathrm{B}_{\mathrm{z}}$ 沿y方向测线分布图。

从测线分布特征可以发现: 测线经过目标正上方 (此 时测线与目标轴向垂直) 时, $\mathrm{B}_{\mathrm{x}}$ 在目标正上方达到峰值, 测线分布关于目标位置总体呈偶对称; $\mathrm{B}_{\mathrm{y}}$ 在目标正上方出 现信号极性反转, 测线分布在目标位置两侧出现波峰和波 谷, 测线分布关于目标位置总体呈奇对称; $\mathrm{B}_{\mathrm{z}}$ 在目标正上 方达到峰值, 测线分布关于目标位置总体呈偶对称; 总磁 感应强度在目标正上方达到峰值, 且关于目标位置总体呈 偶对称。随着测线位置高度的增加, 三轴响应信号幅值逐 渐减小。各分量磁感应强度沿x方向测线分布峰值强度见 表3。

表3 各分量磁感应强度沿y方向测线分布峰值强度。

\begin{tabular}{lllll}
\hline \multirow{2}{*}{ 测线编号 } & \multicolumn{4}{l}{ 各分量测线分布峰值强度(nT) } \\
\cline { 2 - 5 } & $\mathbf{B x}$ & $\mathbf{B y}$ & $\mathbf{B z}$ & MagB \\
\hline LineYInSea & 0.125 & 0.6 & 1.25 & 1.3 \\
LineYInAir_1m & 0.14 & 0.55 & 0.9 & 0.9 \\
LineYInAir_100m & 0.025 & 0.1 & 0.20 & 0.2 \\
\hline
\end{tabular}

通过仿真结果还发现, $13 \mathrm{~ms} \sim 16 \mathrm{~ms}$ 阶段 $\mathrm{Bx} 、 \mathrm{By}$ 和 $\mathrm{Bz}$ 及总磁感应强度信号的测线分布基本一致, 即目标的存在 引起的测线分布异常持续时间较长 (3ms以上), 因此对 于异常信号观测的速度要求较低（采样率较低），关断期 间观测信号的圥余度较高。因此, 测线图绘制及定性解释 时能够进一步降低工作量, 提高效率。

需要注意的是, 沿 $\mathrm{x}$ 方向测线和 $\mathrm{y}$ 方向测线观测结果在 目标物正上方峰值不完全一致, 比如表 2 和表 3 中 $\mathrm{Bx}$ 和 $\mathrm{By}$ 分量, 这可能是仿真模型求解域网格剖分在 $x$ 方向和 $y$ 方向 密度不完全一致, 导致计算测线上结果时出现较大偏差。

\subsection{2. 测面仿真结果及分析}

图17给出了脉冲间歇期目标在海水表面及海底磁感 应强度分布。仿真结果表明, 目标位置正上方的海水表面 及正下方海底出现明显的磁异常, 该异常磁感应强度的大 小能够被当前常用磁场传感器感知, 图磁通门磁力仪及高 温超导量子磁强计。在发射电流峰值为 $5000 \mathrm{kA}$, 发射天线 长度为 $100 \mathrm{~m}$ 时, 对于距离水平发射天线水平偏移距 $707 \mathrm{~m}$ 的处于水下 $100 \mathrm{~m}$ 深的目标能够产生最大 $1.3 \mathrm{nT}$ 的磁异常, 此时目标可以被探测到。

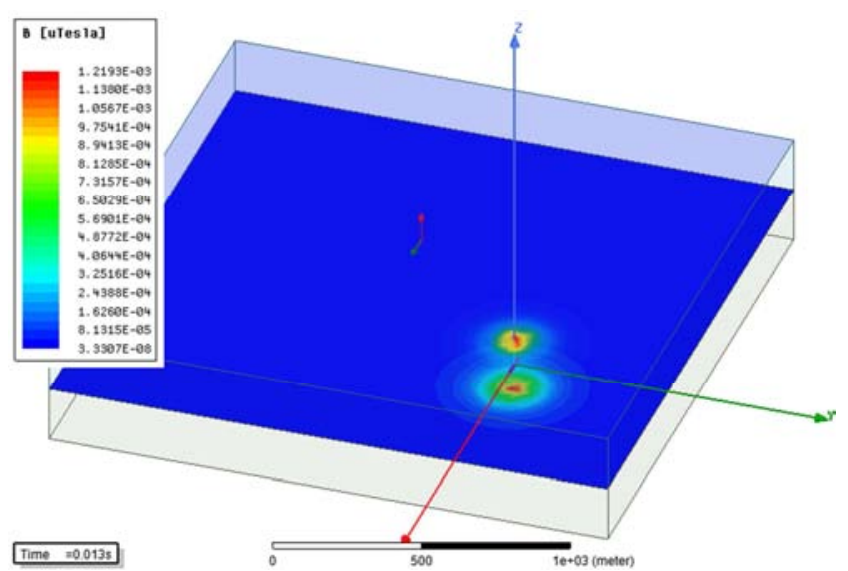

图17 目标在海水表面及海底引起的异常磁感应强度分布, 目标位于水 下 $100 \mathrm{~m}$ 处。

\section{4. 实验验证与分析}

介绍探索性实验的设计思路, 可以对等效缩比实验设 计过程进行简要说明。给出实验设置的参数、场地、目标 物、设备及设备性能说明及相关图表, 给出实验获得的环 境噪声、电流数据、磁场数据, 给出原始数据图和预处理 后的结果图, 对处理后的结果图进行简要分析, 最后落脚 到等效缩比实验验证的探测技术的可能性及对应的信号 水平及设备参数建议。

\section{1. 水下目标主动探测实验}

本课题团队于 2020 年 11 月在浙江舟山进行了水下目 标主动探测缩比验证性实验, 实验地点见图18。实验用各 设备相关信息见表4，相关设备见图19-图22。

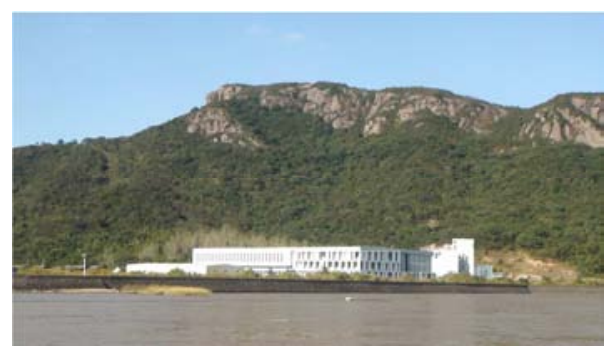

图18 水下目标主动探测实验地点。

实验过程中采用 $5 \mathrm{~m}$ 长的水下水平电性天线, 天线距 离水面约 $0.5 \mathrm{~m}$, 天线与目标距离在 $20 \mathrm{~m}-35 \mathrm{~m}$ 范围可根据需 要进行调整, 目标沉入海水水下 $1.5 \mathrm{~m}$ 。

表4 水下目标主动探测实验各设备信息。

\begin{tabular}{llll}
\hline 序 & \multicolumn{3}{l}{ 实验用设备信息 } \\
\cline { 2 - 4 } 号 & 设备名称 & 设备参数 & 功能用途 \\
\hline \multirow{2}{*}{1} & 电磁场源 & 最大功率 $50 \mathrm{~kW}$, 基频占空可调, & 主动辐射低 \\
& 产生装置 & 最大输出 $100 \mathrm{~A}$ & 频电磁波 \\
2 & 电磁数据 & 三轴同时观测, 6通道采集, & 采集记录监 \\
2 & 接收装置 & $6 \mathrm{pT} / \mathrm{sqrt}(\mathrm{Hz})$ & 测磁场信号 \\
3 & 目标物 & 轴向长 $5 \mathrm{~m}$, 外径 $0.5 \mathrm{~m}$ & - \\
4 & 实验场地 & 长宽高 $53 \mathrm{~m} \times 7 \mathrm{~m} \times 3.3 \mathrm{~m}$ & - \\
\hline
\end{tabular}




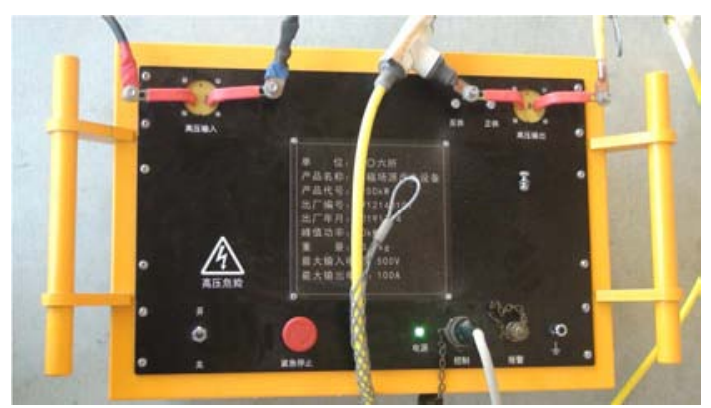

图19 电磁场源产生装置。

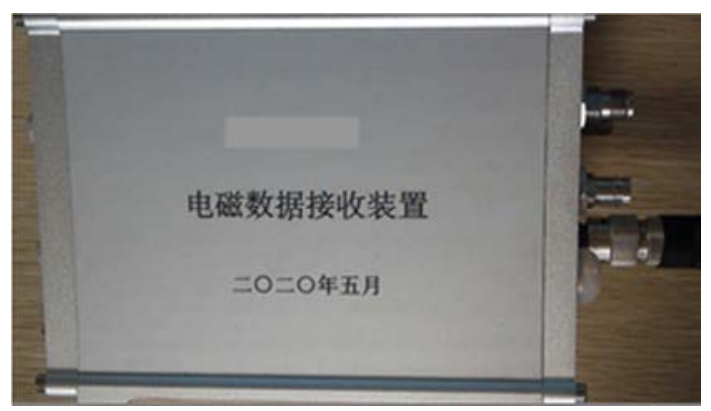

图20 电磁数据接收装置。

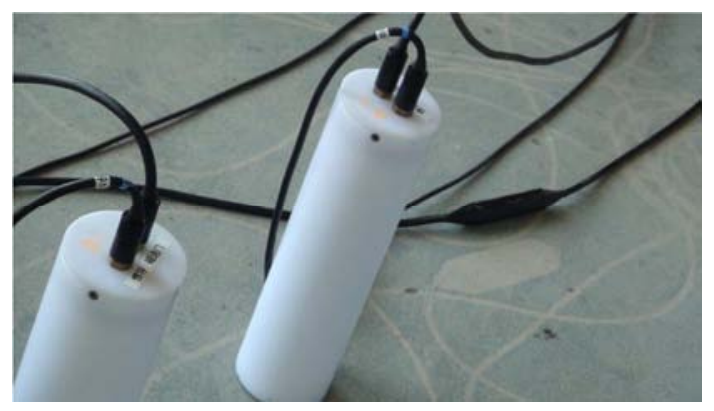

图21 磁场传感器。

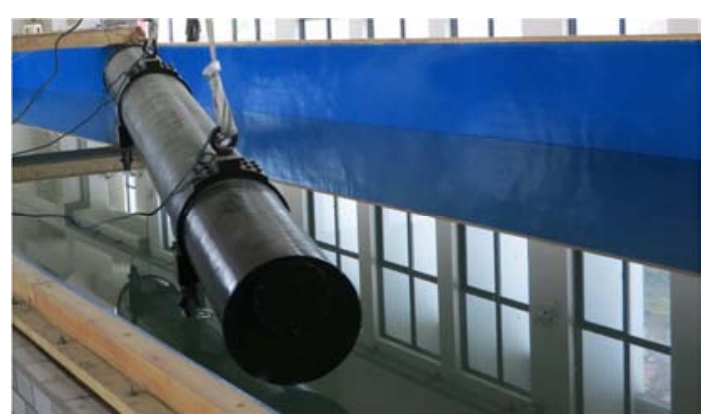

图12 目标物。

实验内容包括背景噪声干扰观测、天线无辐射情况下 目标磁异常观测、天线辐射情况下目标耦合磁信号观测。 基本思路是, 目标磁异常和目标耦合磁信号分别与背景噪 声相减获得主被动模式下目标存在导致的磁场分布异常 对比分析。

实验观测背景磁场干扰强度水平方向分别是 $14 \mathrm{nT}$ 和 $5 \mathrm{nT}$, 垂直方向 $12 \mathrm{nT}$ 。天线辐射电磁波时发射电流强度约 $30 \mathrm{~A}$, 距离发射天线 $25 \mathrm{~m}$ 处水面上方约 $2 \mathrm{~m}$ 处的磁感应强度 信号见图18, 其中红色实线为原始信号, 黑色实线为PCA 后的信号。

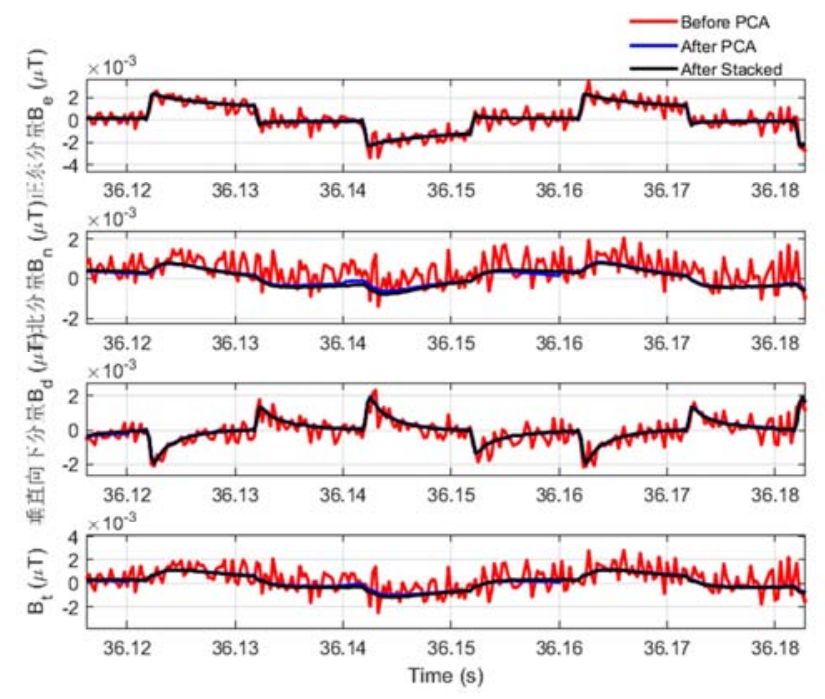

图23 水池上方 $(X=3 m, Y=26 m)$ 处观测各方向磁感应强度信号。

\section{2. 水下目标主动探测实验结果与分析}

\subsection{1. 被动模式目标探测效果}

图 24 给出了目标沿东西方向布置于长边方向 $28 \mathrm{~m}-33 \mathrm{~m}$ 之间时, 目标在水面上方约 $2 \mathrm{~m}$ 高度平面磁图分布。 磁图显示, 被动模式下可以较清晰的显示大约在 $30 \mathrm{~m}-35 \mathrm{~m}$ 之间存在异常。

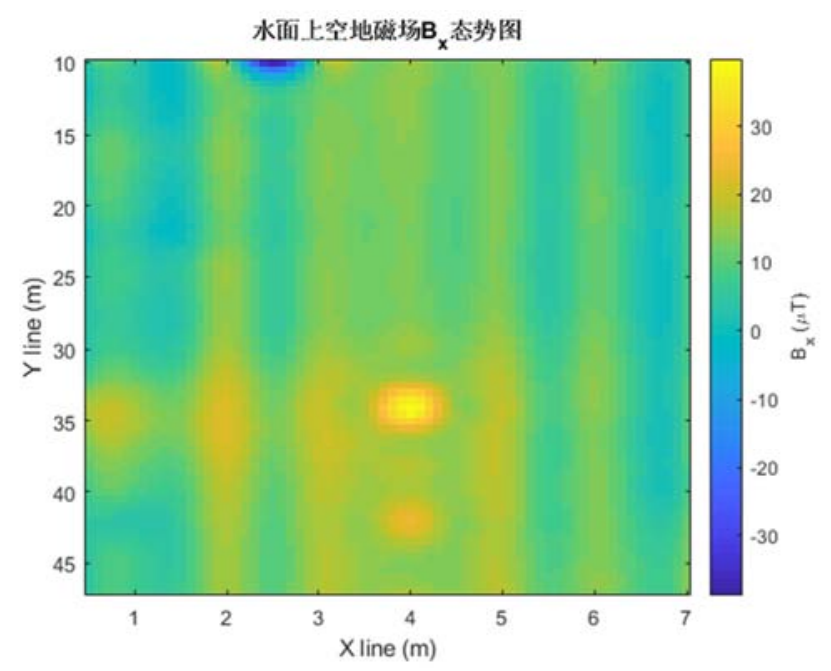

图24 被动模式下, 目标存在引起的 $\mathrm{B}_{\mathrm{x}}$ 分量磁图。

\subsection{2. 主动模式目标探测效果}

图 25 给出了目标沿东西方向布置于长边方向 $28 \mathrm{~m}-33 \mathrm{~m}$ 之间时, 目标在水面上方约 $2 \mathrm{~m}$ 高度平面磁图分布。 磁图显示, 主动模式下可以较清晰的显示大约在 $30 \mathrm{~m}-35 \mathrm{~m}$ 之间存在异常, 并且可以清晰显示出目标位置附近的耦合 磁异常波峰和波谷效应。图26则给出了主动模式下目标耦 合磁感应强度分量 $\mathrm{Bx}$ 在去除地磁场引起的磁异常后的磁 图分布。可以发现, 主动模式相较被动模式对水下目标探 测具有更高的信噪比, 同时可以发现, 联合主被动模式探 测信息可以对干扰去除, 实现对目标的准确探测, 降低虚 警。 


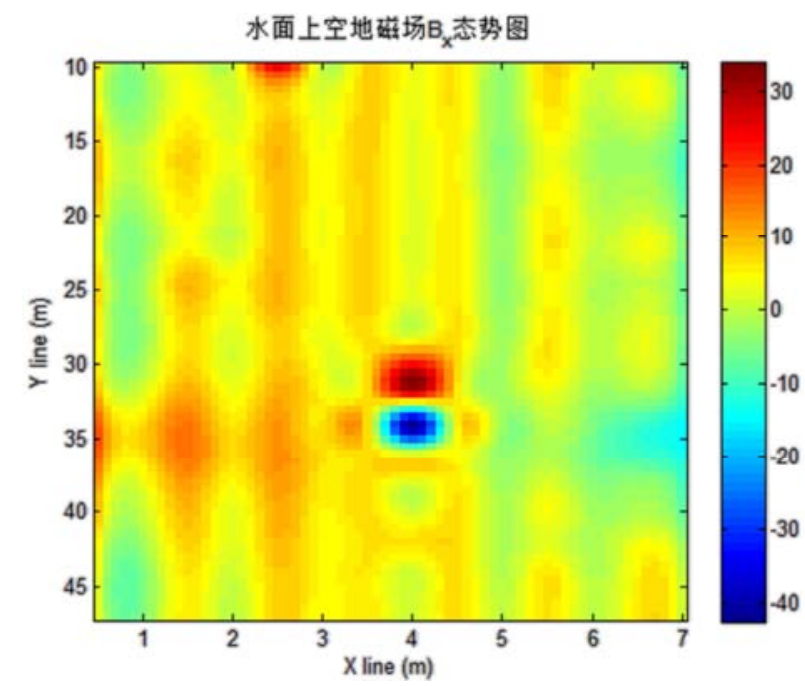

图 25 主动模式下, 目标存在引起的 $\mathrm{B}_{\mathrm{x}}$ 分量磁图。

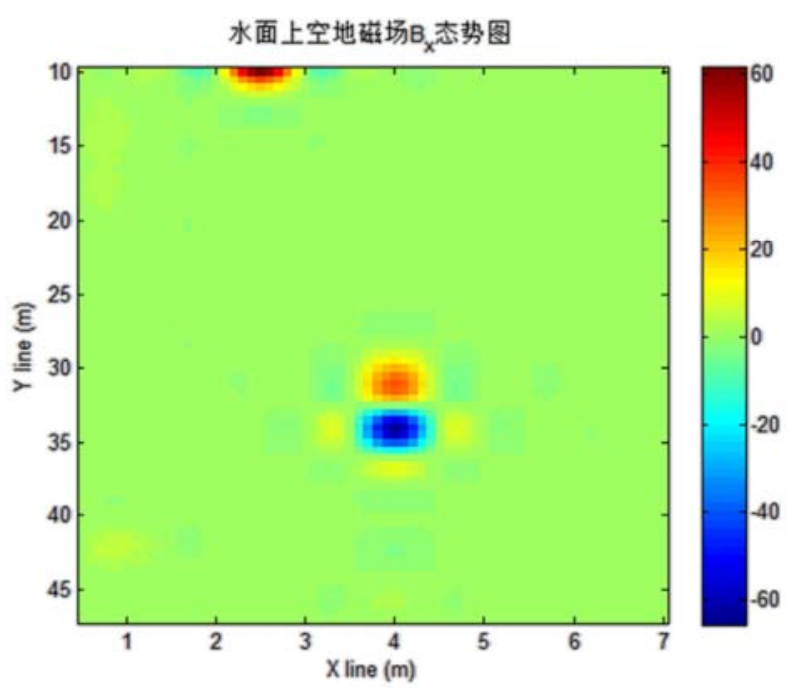

图26 目标存在时, 主动模式下 $B_{x}$ 去除目标磁异常后的磁图。

\section{5. 结论}

为探索主动电磁探测技术应用于水下大型目标探测 定位的可能性及探测模式, 论文通过研究电磁波在海水中 的传播规律及水下偶极天线辐射电磁场的分布规律和性 能对比, 提出了基于超低频(SLF)/极低频(ELF)的人工电磁 场源的水下目标主动电磁探测方法, 利用Ansys Maxwell 建模仿真, 分析目标耦合磁场强度和分布规律, 据此进行 水下目标主动电磁探测系统关键参数设计, 研制了一套水 下目标主动电磁探测样机; 最后, 通过缩比验证实验对水 下目标主动探测方法进行了初步验证。仿真结果表明，基 于SLF/ELF电磁波的主动电磁探测技术能够应用于水下 大型目标的探测。缩比实验结果表明, 根据仿真结果设计 的缩比样机系统参数正确有效。本论文证明了基于 SLF/ELF主动电磁探测技术应用于水下大型目标探测的 可能性, 下一步课题组将从水下天线辐射效率提升优化设 计、传感器性能提升及电磁数据精细处理等方向进一步深 入研究, 为工程样机的性能提升提供技术基础。

\section{参考文献}

[1] 渠晓东. 甚低频/极低频电磁法在目标探测中的应用[D]. 北 京: 中国科学院大学, 2017: 62-64。

[2] 申茂冬. 高温超导全张量磁梯度测量技术研究[D]. 长春: 吉 林大学, 2017。

[3] Ping $\mathrm{Yu}$ et al. The analysis on Marine Controlled-Source Electromagnetic Research Status [J]. IOP Conf. Ser.:Earth and Environmental Science, 2019. 252(5).

[4] 翟景红.海洋聚焦电磁法理论与实验研究[D].2018, 北京: 中 国地质大学(北京), 2018:13-33。

[5] S. Durrani. Air-to-undersea communication with electric dipoles. Antennas and Propagation[J], IRE Transactions on, 1962, 10(5): 524-528.

[6] S. Durrani. Air to undersea communication with magnetic dipoles. Antennas and Propagation [J], IEEE Transactions on, 1964, 12 (4): 464-470.

[7] H. Wang, K. Zheng, K. Yang et al. Electromagnetic field in air produced by a horizontal magnetic dipole immersed in sea: theoretical analysis and experimental results [J]. Antennas and Propagation, IEEE Transactions on, 2014, 62 (9): 4647-4655.

[8] H. Wang, K. Yang, K. Zheng. Electromagnetic field radiated in air from a horizontal/vertical magnetic dipole in sea. Journal of Electromagnetic Waves and Applications, 2015, 29 (7): 858-873.

[9] 王宏䂞.电磁波跨越海-空界面传播特性研究[D].西安：西北 工业大学, 2015:11-17, 32-53。

[10] Shi Z, Liu L, Xiao P, et al. Simulation and Analysis of the effect of ungrounded rectangular loop distributed parameters on TEM response [J]. Journal of Applied Geophysics, 2018, 149.

[11] 时宗洋.半航空瞬变电磁系统探测方法研究[D].北京: 中国 科学院大学, 2018: 41-42。

[12] 王杨婧.水下大型目标的磁探测研究[D].西安: 西安电子科 技大学, 2012。

[13] Nabighian M N. Electromagnetic Methods in Applied Geophysics: Volume 1, Theory [M]. Society of Exploration Geophysicists, 1991。

[14] 渠晓东.甚低频/极低频电磁法在目标探测中的应用[D]. 北 京: 中国科学院大学, 2017: 62-64。

[15] Kaufman A A, Keller G V. Frequency and transient soundings [M]. Springer, 1983。

[16] NIGEL EDWARDS. MARINE CONTROLLED SOURCE ELECTROMAGNETICS: PRINCIPLES, METHODOLOGIES, FUTURE COMMERCIAL APPLICATIONS [J]. Surveys in Geophysics, 2005, 26: 675-700.

[17] Rong Liu, Jianin Liu, Jianxin Wang, Zhuo Liu, Rongwen Guo. 1D EM response modeling with arbitrary source-receiver geometry based on vector potential and its implementation in $\begin{array}{lllll}\text { Matlab } & {[\mathrm{J}] .} & \text { Geophysics, } & 2020 . & 85\end{array}$ F27-F38.DOI:10.1190/geo2019-0224.1. 
[18] KerryKey, OCCAM1DCSEM An Open-Source Inversion Program for Generating Smooth 1D Models from Controlled-Source Electromagnetic and Magnetotelluric Data [R]. San Diego: Scripps Institution of Oceanography, University of California, 2011.
[19] Andrew Pethick, Planning and 4D Visualisation of the marine controlled source electromagnetic method [D]. Australia: Department of Exploration Geophysics, Curtin University of Technology, 2008. 